\title{
Interview with Akshay Venkatesh
}

Ulf Persson (Chalmers University of Technology, Göteborg, Sweden), Editor of the EMS Newsletter

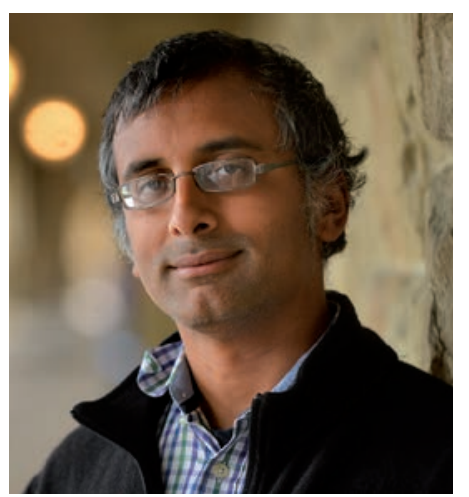

UP: As usual, my first stupid question is whether you were surprised to receive the Fields Medal? AV: Honestly, I was.

Are you sure? How did you find out about it?

I got an email from Mori asking me to get in touch with him on Skype.

Photo by Rod Searcy.

Do you think that being a Fields Medallist will change your life? You are now a kind of movie-star celebrity but that status may well evaporate after the congress is over.

It is pleasant while it lasts but I hope that I will not suffer from additional pressure of expectation and will be able to carry on in my normal, carefree way.

You were a prodigy of sorts, participating in all kinds of mathematical Olympiads even before you were in your teens. By the way, when did you go to university?

At 13.

This is young - exceptionally so, I would say. So you were a real prodigy, which leads me to the question of competition in mathematics. When you first encounter mathematics at school, this is the subject most adapted for excelling in and showing that you are smart. But when did you discover that mathematics was a subject interesting in itself?

As to your original question, let me put it this way: I like the social aspect of mathematics, working and interacting with other people. On the other hand, I would hate to be in the position of being one of two people working on the same problem and see who gets there first. I want to be in a more relaxed situation. As to your second question, I guess it was at university when I first encountered the richness of mathematics...

...One should not forget that you were only 13 at the time. Please go ahead!

I learned a lot at the time by reading a huge number of books and articles.

How do you read an article?

I never actually recall reading an article from cover to cover. I tend only to skim through them and look out for what is interesting.

This is a common experience among the Fields Medallists that I have interviewed. In a way, writers of articles waste their time constructing carefully structured 
narratives. It is the same thing with lectures: you let it just pass and then once or twice you catch something of interest and decide to take it home with you. But, of course, even if the structured narrative of a lecture appears superfluous in retrospect, you would never enjoy just random mutterings by the lecturer throwing out unrelated titbits of facts and ideas at you (although I have 'suffered' through such lectures). Even if you just pick a few of the fruits, you want them nicely presented. By the way, your talk was really very excellent.

(beaming) You think so?

Definitely. You dispensed with tedious details and elaborate reasoning and precise statements without being vague and vacuous (as such lectures otherwise often turn out to be). I particularly liked your way of suggesting what a reciprocity theorem is really about, namely connecting primes with similar behaviour in specific situations. Your remarks on Hecke operators were also instructive. Such remarks are usually omitted in standard presentations but may be conveyed in private conversations. Speaking a bit metaphorically, in a lecture you want to know why a certain computation is being made but not see it performed. When you give lectures to students in a lecture course, going through computations may be justified but never to colleagues. You did it very well.

Thank you. I actually worried a bit. I have never given a talk to such a large and varied audience before.

It went very well. But, of course, you need to know some mathematics. I do not think that my wife would have been as enraptured. If you are not familiar with primes and have never heard about the Langlands programme, you would not get much out of it, if you do not mind me saying.

Of course not.

You mentioned in the video that you liked manipulating numbers when you were a child.

Not only as a child; I still do as an adult.

It is hard to be a mathematician, especially a number theorist, and not know that 2 is a prime but, in principle, you could go through life as a mathematician without caring whether 23 is a prime or not. We have the famous Grothendieck prime 27. I guess you care and, like Ramanujan, individual numbers as such are friends to you. I might not go as far as that and I certainly would not pretend to rival Ramanujan. But it is true, if you devote your life to numbers, you better love them as individuals; that is only human.

Would you care to elaborate more concretely what it means to be friends with numbers and how it may manifest itself not just in mathematics but in everyday life?

I would rather point out that for a number theorist, especially nowadays, there are richer objects than mere numbers to be friends with. As examples, I can mention the cubic number field of discriminant -23 or the elliptic curve of conductor 11 . Both are very pleasant company and it is also very useful to have them at your fingertips. But, I have to admit that many other such examples I have outsourced to my laptop. There is a limit to how many intimate friends you can have.

Speaking about laptops, I guess you came into contact with computers very early.

No. In fact, it was not until eight years after my PhD that I discovered computers.

Really? Yet, being so young you must have grown up with them, unlike my generation.

It is true but I did not, strange as it may appear, connect computers with mathematics. Number theory is taught and conducted in a very abstract manner and you do not get your hands dirty caring about whether 23 is a prime - to do so is looked down upon. But computers really meant a revolution to me; it fundamentally changed the way I think of mathematics - especially number theory.

And ties up with your childhood pleasure of manipulating individual numbers.

Of course. Making experimental calculations makes the subject so much more tangible. It is a great experience to have a theorem of yours numerically verified. It makes matter almost uncannily real.

It does confirm the Platonic nature of mathematics: that although mathematics is done by humans, the facts and results are independent of us; we just throw some light on them and they do not care an iota about us. Also, a numerical test tends to be more persuasive than just going through the arguments. It is indeed mechanical and unsentimental, impervious to wishful thinking. I want to come back to that later. Sorry for the interruption.

More seriously, doing computer experiments really guides you and allows you to abandon unfruitful avenues of research.

In geometry and also in analysis, to some extent, you can acquire a visual intuition; this is not the case with numbers, hence you lack the same immediate overview that the visual sense supplies. Thus, such experiments are invaluable; people like Gauss and Euler and even Riemann calculated a lot...

...And by hand to boot. This is really impressive. Once my laptop crashed and I was forced to do a long computation by hand. Isn't it tedious, and how many mistakes do you make? In fact, when you program, you make a lot of mistakes, which are, of course, pitilessly shown up by the compiler. It makes me wonder how many mistakes there must be in a run-of-the-mill paper.

This has struck me too. I know I am being cynical but that is the privilege of my age, maybe the only one; in most cases, it does not make too much of a difference most papers are not read.

But it is different. Mistakes in programming are seldom conceptual and can, in most cases, be easily fixed but of course are catastrophic to the running of the program (a 
misplaced comma causes havoc). A mathematical proof is much more stable, unless of course it is very formal.

Programming is a very relaxing activity, in my opinion; you never get stuck as you do in mathematics. You are not alone; through the compiler and test runs, you can actually converse with the computer and, if things do not work out, you always feel that you are within an epsilon of resolving the problem and you can keep on for hours. It is very seductive.

But when it comes to mathematics, I can always rely on co-workers with new ideas when I get stuck. Anyway, I repeat: actual computations are essential to number theory.

May I be so bold as to presume that the discovery of computer-based computations gave you a new lease on mathematics, making it even more exciting because of that connection?

Yes, indeed.

Talking about formal proofs and reducing them to some kind of super computation invites the issue of AI, which has not so far made any major intrusions into mathematics as it has in games like chess and go. What is your opinion on computer-assisted proofs (not just proof checking), which should in principle be within reach soon (although I guess it is a labour-intensive task to translate actual proofs into formal strings amenable to manipulation by computers, and I am personally a bit suspicious, as there are many subtle forms of reasoning we employ in proofs and it is not clear to me that we can exhaust them in advance), and also the construction of actual proofs. I am sorry for being long-winded but if we think of the ultimate, it would mean that we could type in a conjecture and then the computer decides whether it is true or not. Would that not kill mathematics; there would be no fun at all. It is the road that matters and not the destination. But there are certain philosophers of AI who seriously claim that mathematicians are wasting their time trying to prove theorems; instead, they should devise theorem-proving programs. This reveals a deep misunderstanding of the nature of mathematics and what makes mathematicians tick.

If computers come up with long proofs that we humans cannot understand, just as they come up with opaque moves in chess and go, it is not much good to me.

Yes, what would be the point?

I would not say that it would be pointless. That would be too categorical a statement.

Still, much of mathematical activity consists of overcoming technical difficulties; this is where the professional shows his mettle. Without those struggles, there would be no appreciation of new concepts, nor would they develop in the first place because nothing comes out of nothing.

There you have a point of course. And I could elaborate on what I really mean but I am sorry - I have a lunch date coming up soon, which I do not want to miss, so I have not enough time. Fire again!

Ideas are the most important in mathematics but, unlike theorems, which can be formulated precisely and provide a stepping stone, an idea cannot be formulated; it has to be inferred, often by an example in which it resides, hidden and to be unfolded by the reader. A specific theorem is just one of many ways an idea can be expressed. To use a theorem without appreciating the guiding principle is cheating; usually what you need is not necessarily encapsulated in the formulation of the theorem but can be accessible through the idea. My question is whether you have been cheating, taking results on trust.

I have to admit that I have done so at times. For example, I make use of results from $p$-adic Hodge theory and unfortunately I have little understanding of the proofs. I try my best to avoid this situation. But I see your point. As mathematics becomes more and more "big science" where there are teams working together, such things will become inevitable and also a bit sad.

Yes. This is a development I see coming and it is not welcome. Would you have preferred to be a mathematician of the past?

There would have been many advantages, one being that you could be much more general and not as specialised as you are forced to be today (and even more so I fear in the future). I have studied some of the mathematicians of the 18th and 19th centuries and have been very impressed with what they were able to do with much less technology and such primitive notions. But now I really have to leave and meet Harald.

\section{We are not finished yet!}

Okay. I can give you some more time but I am sorry - it cannot be much.

One speaks about problem solvers and those who like Grothendieck, wanting to build theories and understand things in some "functorial" way. Not only should a theorem be proved, it should also be proved in a natural and inevitable way out of the theory in which it dwelt. Grothendieck hated tricks.

I must say that I am neither. I do not aim for a general understanding - that is too ambitious for me. I am more modest; I am just happy to come across objects that are congenial to me, with a lot of rich structure to explore. But now I really must be going.

Do you mind if I tag along?

Not all the way to lunch though; I have some things I want to discuss with Harald.

(approaching) But that is Helfgott. I did one on him a few years ago.

He survived.

Yes, and is still smiling. Hello Harald... See you. It has been fun talking to you. 